\title{
Growth and Patterning of Pt Nanowires on Silicon Substrates
}

\author{
Eric P. Lee and Younan Xia $(\bowtie)$ \\ Department of Biomedical Engineering, Washington University, St. Louis, Missouri 63130, USA \\ Received: 27 May 2008/ Revised: 23 June 2008/Accepted: 25 June 2008 \\ CTsinghua Press and Springer-Verlag 2008
}

\begin{abstract}
This paper describes a simple procedure for growing dense films of Pt nanowires directly on silicon substrates by modifying the surface through chemical or physical means. In the former, a self-assembled monolayer of (3-mercaptopropyl)-trimethoxysilane (MPTMS) is applied which can strongly bind $\mathrm{Pt}(0)$ nuclei to the surface through $\mathrm{Pt}-\mathrm{S}$ linkages. Once attached, the $\mathrm{Pt}(0)$ nuclei can act as catalytic sites for the growth of Pt nanowires along the $\langle 111\rangle$ direction. Alternately, relief features are physically created on the surface in order to generate nucleation and binding sites for $\mathrm{Pt}(0)$ nuclei, due to the higher free energy associated with a rough surface. Additionally, Pt nanowires have been successfully produced in well-defined patterns by scouring grooves on the silicon surface or by photochemically patterning the MPTMS monolayers with a shadow mask. We have also measured the electrochemical properties of these immobilized or patterned Pt nanowires. The results provide an effective route to producing dense films of Pt nanowires with high surface areas for various electrochemical applications.
\end{abstract}

\section{KEYWORDS}

Platinum nanowires, silicon, patterned array, high surface area, electrochemistry

Noble-metal nanostructures have been extensively studied in recent years because of their importance in various applications, such as catalysis, fuel cells, and high performance electrochemical sensors [1-3]. Platinum nanostructures have received considerable interest, as this metal is known to be one of the most important catalysts for many industrial processes, including hydrogenation reactions, three-way automobile catalytic conversion, and especially the hydrogen or methanol oxidation reaction (MOR) and the oxygen reduction reaction (ORR) in fuel cells [4-9]. It is generally accepted that both catalytic efficiency and selectivity are highly dependent on the size and shape of the platinum material. Therefore, a great deal of research has been devoted to the synthesis of Pt nanostructures with well-controlled morphologies [10-14].

In our previous work [15, 16], we demonstrated a simple and practical method for growing $\mathrm{Pt}$ nanowires as densely packed arrays supported on a number of templates. This synthesis is remarkably straightforward and versatile, generating Pt nanowires with extremely high surface areas and structural uniformity. Consequently, these Pt nanostructures are expected to be ideal candidates for use as catalysts in various industrial applications, especially fuel cells. So far, we have only demonstrated the growth of $\mathrm{Pt}$ nanowires on ceramic and polymeric microbeads

Address correspondence to xia@biomed.wustl.edu 
and on precious metal meshes made of $\mathrm{Pt}$ or $\mathrm{W}$. While each support provides a suitable substrate for Pt nanowire growth, their cost and functionality are still rather limited for industrial applications. Herein we demonstrate the use of silicon as an alternative support, which is more desirable for commercial use.

Silicon is beginning to be used as a catalyst support in various micro power fuel cell designs due to its conductive nature and low cost. Although silicon is not considered to be an ideal current collector, it is expected to effectively draw current from the reaction region of a fuel cell [17]. In recent years, a new class of small reactors, called micro-fuel cells, has emerged as an alternative power source for portable electronic devices, and in this regard, silicon has become known as one of the most promising support materials for the development of micro-fuel cells $[18,19]$. One reason for the appeal of silicon is that the established microelectronic fabrication technology provides a cost-effective, reproducible, and well-controlled technique for the miniaturization and development of novel miniature fuel cells [20]. Silicon can also be easily coated with a catalyst which converts the otherwise catalytically inactive silicon into a functional electrode. The most common method involves sputtering a thin layer of carbon onto the silicon substrate followed by sputtering or electrodepositing $\mathrm{Pt}$, or another active catalyst, on the surface [21]. The sputtering method, although simple, provides a catalyst layer with a relatively low electrochemically active surface area. Here we describe a simple, bench-top, wet chemical method for integrating a high active surface area catalyst layer of Pt nanowires on a silicon substrate with a flat, contoured, or patterned surface.

The work presented in this paper is novel and exciting as there are very few demonstrations regarding the synthesis of nanowires on solid supports, and even fewer demonstrations for patterned forms [22-24]. Although ordered arrays of nanoparticles on oxide supports have already been produced [25], in general bottom-up approaches are not easily adapted to generate controllable patterns according to the application requirements, and this is even less so for the assembly of one-dimensional (1-D) nanostructures [26]. Furthermore, there is no guarantee that a solution-phase method, the simplest and most robust technique for bottom-up synthesis, can be extended to form nanowires as a supported coating. We have tackled this challenge and successfully patterned large areas of silicon with Pt nanowires. This feat can potentially be of benefit in the electronic, sensing, and catalytic industries as it is becoming more likely that the future of these applications will require integration of numerous 1-D nanostructures through assembly, patterning, and alignment on solid supports over large areas [27].

In order to grow $\mathrm{Pt}$ nanowires as a dense thin film on silicon, it is critical to functionalize the surface chemically or texture the surface physically. Without any modification, a polished silicon wafer has a chemically inert and featureless surface (see the inset of Fig. 1(a)). As such, it provides no aid to the nucleation and growth of Pt nanowires. To chemically prepare silicon for the growth of $\mathrm{Pt}$ nanowires, a typical experiment began by dip-coating a clean silicon substrate in a $0.5 \mathrm{mmol} / \mathrm{L}$ solution of (3-mercaptopropyl)-trimethoxysilane (MPTMS). After washing the functionalized substrate with copious amounts of ethanol and drying under a continuous stream of air, it was added to an iron-mediated polyol reduction bath and heated at $110{ }^{\circ} \mathrm{C}$. In a typical synthesis, $1 \mathrm{~mL}$ of $\mathrm{H}_{2} \mathrm{PtCl}_{6}$ solution in ethylene glycol $(80 \mathrm{mmol} / \mathrm{L})$ was added into the solution along with $1 \mathrm{~mL}$ of poly(vinyl pyrrolidone) (PVP) solution in ethylene glycol $(400 \mathrm{mmol} / \mathrm{L}$, in terms of the repeat unit). A trace amount of $\mathrm{FeCl}_{3}$ was also added to serve as an oxidative etchant for $\mathrm{Pt}(0)$ (see Experimental section for details). Once initiated, the Pt nanowires could continually grow until the supply of $\mathrm{Pt}(0)$ atoms was exhausted [15, 16]. Due to the presence of thiol moieties (from MPTMS), the Pt nanowires could nucleate and grow from the silicon surface, resulting in a dense film of Pt nanowires across the entire substrate surface. After reacting for $18 \mathrm{~h}$, black aggregates began to appear on the silicon substrate, indicating the formation of a $\mathrm{Pt}$ nanowire coating. As shown in Fig. 1(a), the array of Pt nanowires is dense and continuous. The key to this synthesis is the strong propersity of the exposed surface of Pt nanoparticles to bind to the terminal thiol group of MPTMS [28-31]. It is known that the 
$\mathrm{H}_{2} \mathrm{PtCl}_{6}$ precursor is initially reduced to a stable $\mathrm{Pt}(\mathrm{II})$ species, as an intermediate, when heated to $110{ }^{\circ} \mathrm{C}$ in ethylene glycol [10]. Continued heating of the intermediate species at $110^{\circ} \mathrm{C}$ results in the formation of $\mathrm{Pt}(0)$ nuclei, which chemisorb onto the MPTMS monolayer through the formation of strong $\mathrm{Pt}^{-}$ $S$ linkages [28-31]. Once attached, the $\mathrm{Pt}(0)$ nuclei serve as catalytic seeds for the anisotropic growth of Pt nanowires. The use of metal nanoparticles as catalytic seeds for growing Pt nanowires has been previously demonstrated by our group for a number of dielectric and metal substrates $[15,16]$. Here we show that this seeding process can also be applied to silicon substrates. As shown in Fig. 1(b), the Pt nanowires are single crystals with the growth direction along the $\langle 111\rangle$ axis. Furthermore, this growth process can be readily extended to silicon substrates with contoured or pitted surfaces to form coatings of Pt nanowires conforming to the surface contours. Figure 1(c) shows an scanning electron microscopy (SEM) image of a silicon substrate with a patterned array of $5 \mu \mathrm{m}$ pits produced by microcontact printing and selective etching. To prepare the substrate for Pt nanowire growth, the surface was functionalized with MPTMS by dip-coating, followed by extensive rinsing with ethanol and drying under a continuous stream of air. By varying the concentration of $\mathrm{H}_{2} \mathrm{PtCl}_{6}$ introduced into the iron-mediated polyol synthesis, the density of Pt nanowires could be controlled: use of higher concentrations of $\mathrm{H}_{2} \mathrm{PtCl}_{6}$ results in an increased density of Pt nanowires. In Fig. 1(d), an $80 \mathrm{mmol} / \mathrm{L}$ solution of $\mathrm{H}_{2} \mathrm{PtCl}_{6}$ was used, and the inset confirms the growth of $\mathrm{Pt}$ nanowires.

Another approach to growing $\mathrm{Pt}$ nanowire films on silicon is to create textured surfaces, which sufficiently promote the nucleation of $\mathrm{Pt}(0)$ atoms and subsequently induce the formation of $\mathrm{Pt}$ nanowires as a deposited thin film. Figure 2(a) shows the SEM image of the unpolished side of a silicon wafer, which is very rough on the surface. As seen in Fig. 2(b), Pt nanowires nucleated and grew from the rough surface during the iron-mediated polyol synthesis. The inset confirms that the deposits growing on the uneven surface are in fact Pt nanowires. We suspect that these Pt nanowires had mainly nucleated at defect sites. It has been shown in other studies that

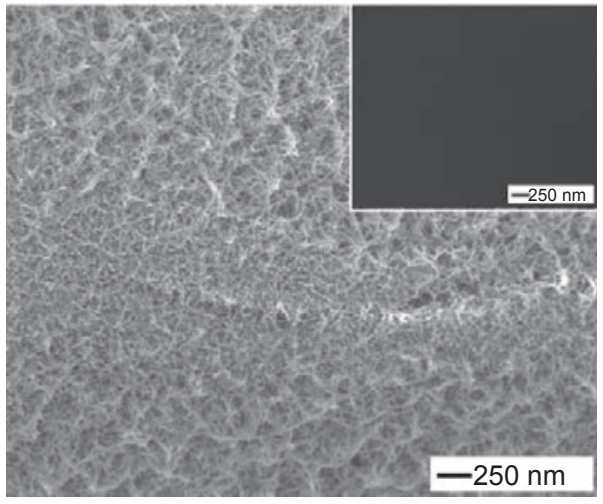

(a)

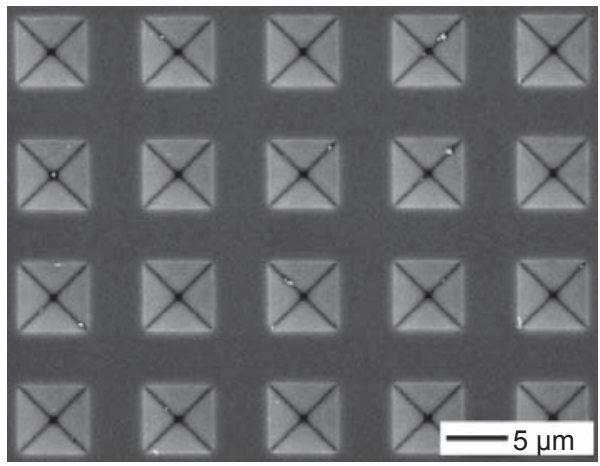

(c)

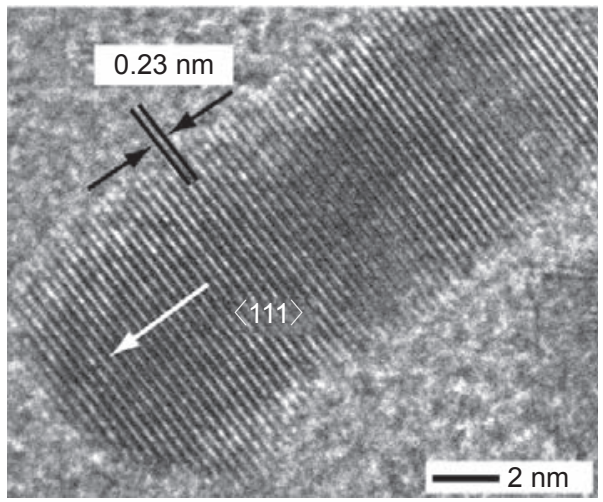

(b)

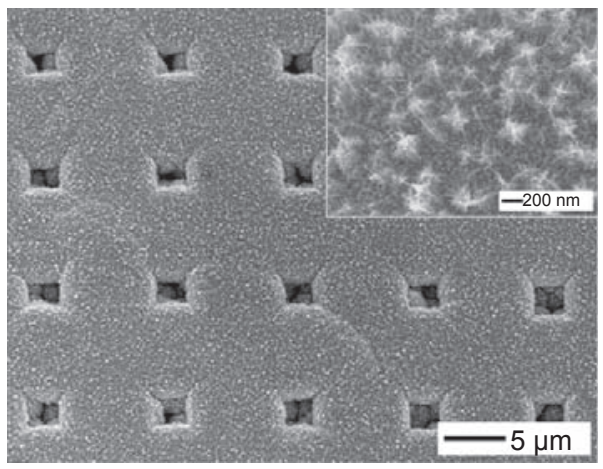

(d)
Figure 1 (a) SEM image showing a dense film of Pt nanowires on the surface of a polished silicon substrate, which was modified with (3-mercaptopropyl)-trimethoxysilane (MPTMS) prior to its introduction into the iron-mediated polyol synthesis; the inset shows a pristine polished silicon substrate, which exhibits a featureless surface. (b) HRTEM image of an individual Pt nanowire released from the sample in (a). The image shows that the nanowire is single crystalline. The fringe spacing of $0.23 \mathrm{~nm}$ corresponds to the interplanar separation between the $\{111\}$ planes, implying that the growth direction of the nanowire is along the $\langle 111\rangle$ axis. (c), (d) SEM images of a pitted silicon substrate before and after growing Pt nanowires on the MPTMS-functionalized surface. The pitted surface was fabricated by patterning and then anisotropic etching. The Pt nanowires were grown from a solution containing $80 \mathrm{mmol} / \mathrm{L} \mathrm{H}_{2} \mathrm{PtCl}_{6}$ 


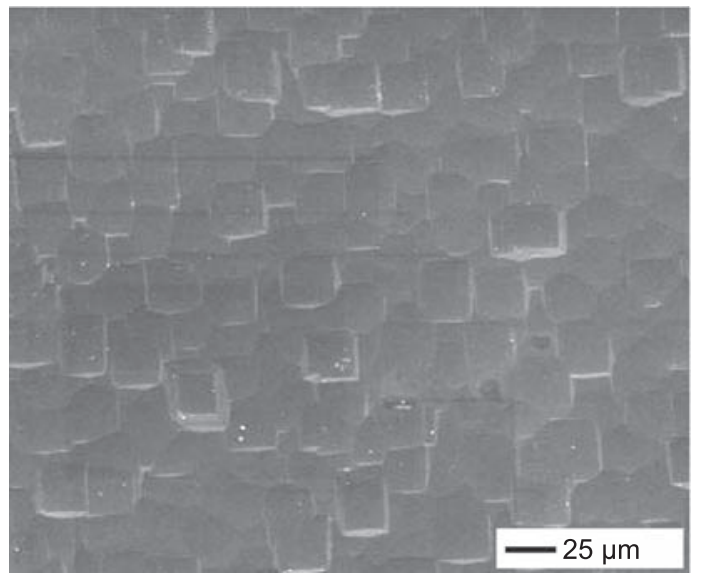

(a)

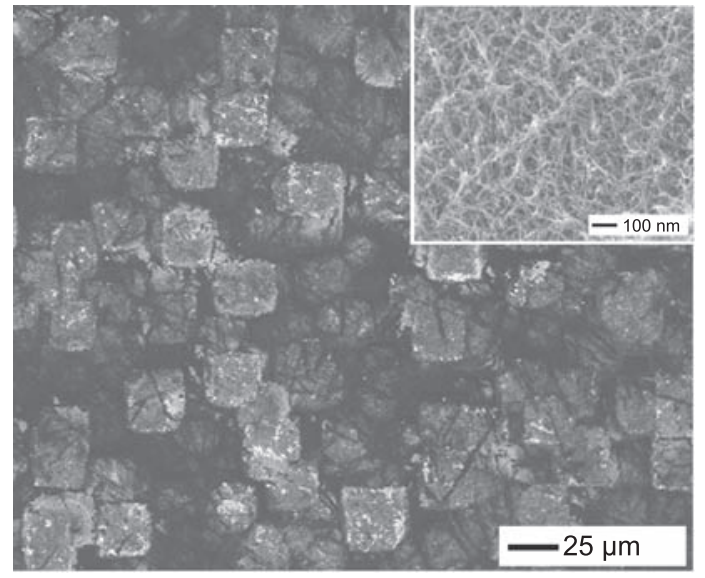

(b)

Figure 2 SEM images of the unpolished side of a silicon wafer (a) before and (b) after Pt nanowires were grown on the surface. The textured features on the unpolished surface can act as nucleation sites for the growth of Pt nanowires, as seen in (b). The inset shows a highmagnification image of the nanowires grown on the rough surface

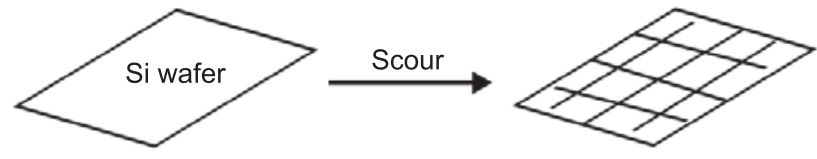

(a)

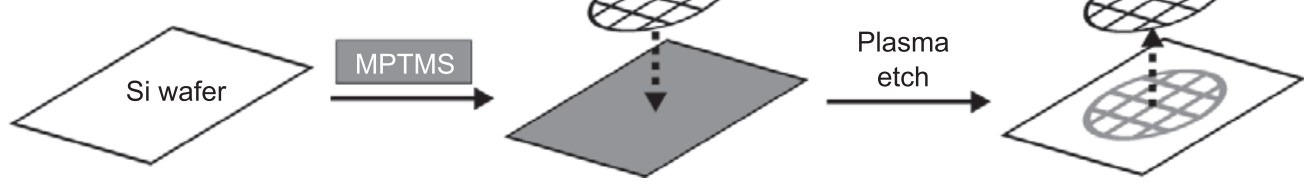

(b)

Figure 3 Schematic outlines of the major steps in generating functional patterns on the surface of silicon substrates. In scheme (a) a clean silicon substrate is patterned with crossing lines by scouring the surface with a diamond cutter. In the first step of scheme (b), a clean silicon substrate was treated with MPTMS by dipcoating, followed by washing with a high volume of ethanol and drying with a continuous flow of air. After functionalizing, a copper grid was placed over the surface as a shadow mask and placed into a plasma cleaner for selective etching. After etching, the regions protected by the shadow, which was cast by the copper grid, remained functionalized while the exposed regions were stripped of MPTMS highly irregular and rough surfaces can serve as primary nucleation sites for the growth of metal nanostructures. This is a result of the higher surface energies associated with the rough features, which significantly lower the barrier for nucleation [32, 33].

By modifying silicon surfaces through chemical functionalization or texturing, we have also demonstrated that Pt nanowires could be grown onto surfaces in well-controlled patterns. Figure 3 illustrates two procedures for creating functional patterns. In the first method (Fig. 3(a)), regions of high surface roughness are created by simply scouring lines across the polished side of a silicon wafer with a diamond cutter [34]. This generates grooves with highly irregular features, which can act as nucleation sites. In the second method (Fig. 3(b)), the silicon surface is first treated with MPTMS by dip-coating, followed by immediate washing with ethanol and drying with a continuous flow of air. Once functionalized, a copper grid was taped over the functionalized surface to serve as a shadow mask. After the sample was exposed to a plasma etching at $100 \mathrm{~W}$ for $10 \mathrm{~min}$, regions in which the copper grid did not cast a shadow were etched by plasma, leaving a checkered pattern in the MPTMS monolayer. As indicated earlier, the regions still functionalized with MPTMS have a high selectivity for binding $\operatorname{Pt}(0)$ nuclei and serve as catalytic sites for the anisotropic growth of Pt nanowires. Figure 4 shows SEM images of the resulting Pt nanowires grown onto the patterned silicon surfaces. Figures 4(a), (b) show SEM images of $\mathrm{Pt}$ nanowires growing mostly from the grooves created by scouring the surface. Figure 4(b) shows that a dense coating was formed at the grooves, and the inset confirms that the coating is composed of nanowires. Figures 4(c), 4(d) show SEM images of Pt nanowires growing on the MPTMS-functionalized surface. The regions 
outside the checkered pattern are mostly bare due to the removal of MPTMS by plasma etching. Figure 4(d) shows a higher magnification image of the boxed region in Fig. 4(c), with an inset confirming that a dense array of nanowires was formed.

To determine whether the Pt nanowires supported on silicon substrates could be used as electrodes, the redox behavior of ferricyanide ions was used as an electrochemical probe to determine the performance of each sample. Figure 5(a) shows cyclic voltammograms comparing the activities of silicon with three different surfaces: bare silicon, a patterned array of $\mathrm{Pt}$ nanowires, and a continuous film of Pt nanowires. All the samples were prepared on 0.5 $\mathrm{cm} \times 0.5 \mathrm{~cm}$ silicon substrates and all the electrochemical experiments were performed

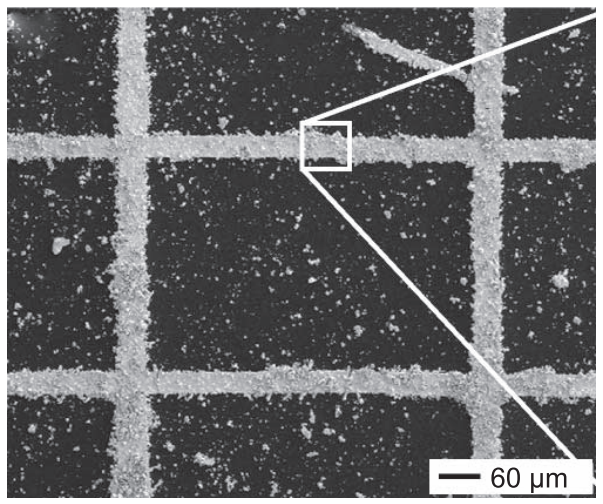

(a)

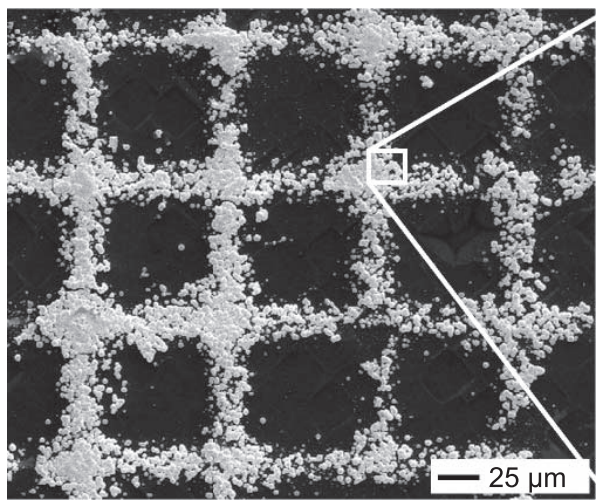

(c)

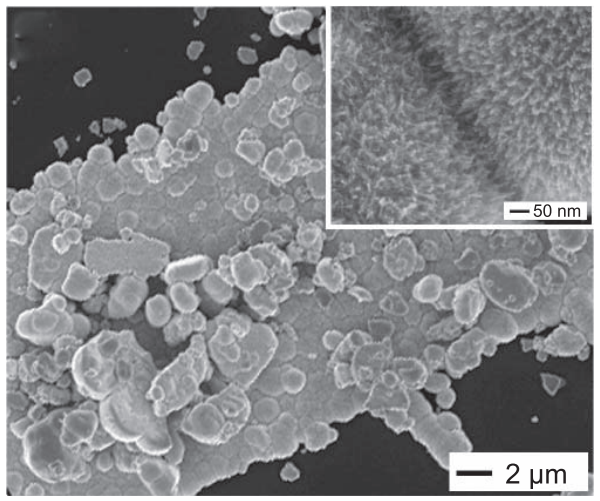

(b)

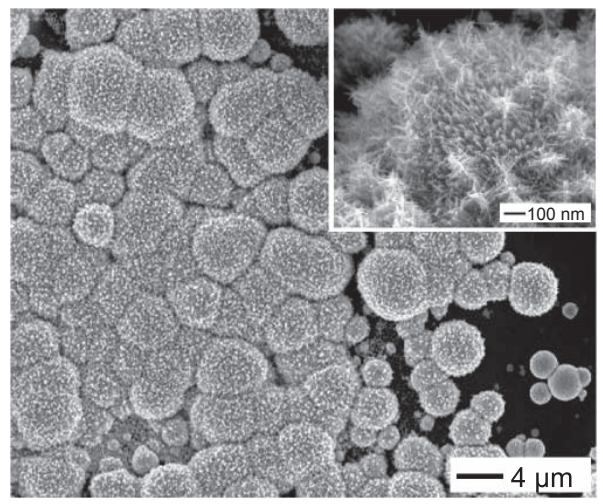

(d)

Figure 4 SEM images of Pt nanowires grown on polished silicon substrates, which had been patterned by either scouring (a), (b) or by etching with a shadow mask (c), (d). (a), (b) A dense array of Pt nanowires was grown in the grooves generated by scouring the substrate. The inset in (b) shows a high-magnification image of the Pt nanowires. (c), (d) Pt nanowires growing from regions functionalized with MPTMS. The patterns were generated by initially functionalizing the surface with MPTMS, followed by plasma etching through a copper grid. The inset in (d) shows a highmagnification image of the Pt nanowires

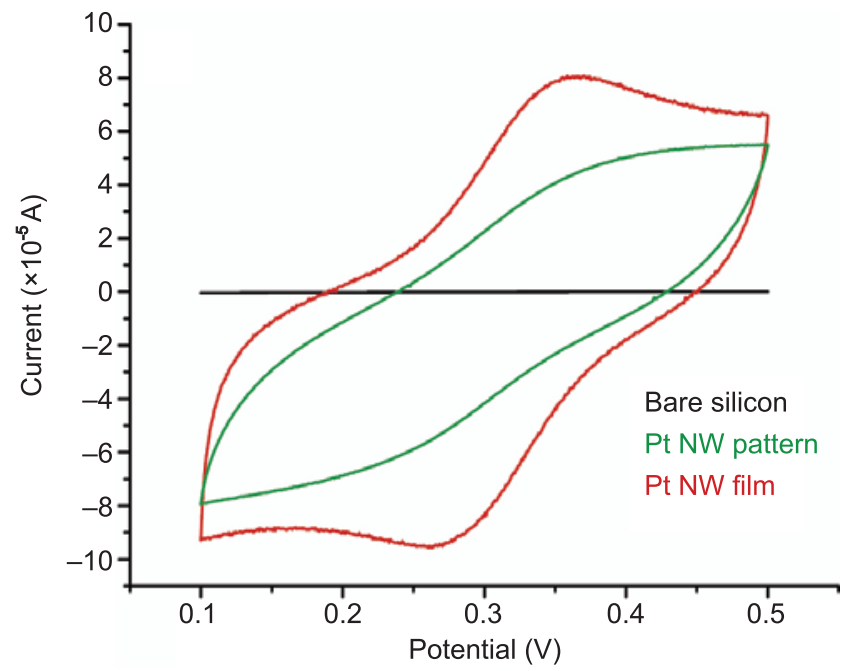

(a)

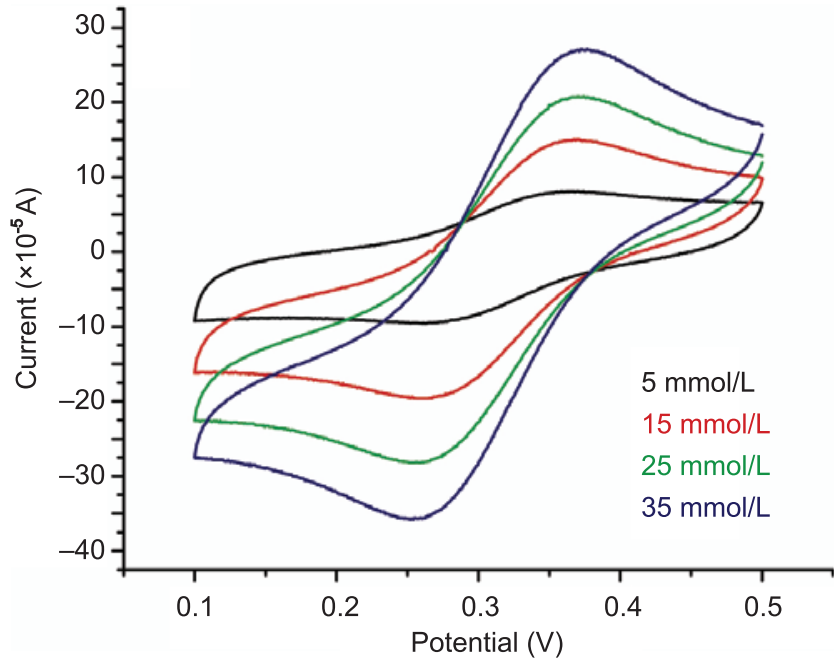

(b)

Figure 5 Cyclic voltammetric curves of silicon substrates whose surface are covered by Pt nanowires as a dense film and a patterned array, performed with a sweep rate of $40 \mathrm{mV} / \mathrm{s}$ and $1 \mathrm{~mol} / \mathrm{L} \mathrm{KCl}$ as the supporting electrolyte. (a) A comparison between different working electrodes: bare silicon, a patterned array of Pt nanowires, and a continuous film of Pt nanowires. The CV curves were performed using $5 \mathrm{mmol} / \mathrm{L}$ ferricyanide solution. (b) Voltammetric responses of a Pt nanowire film sample at different ferricyanide concentrations ranging from $5 \mathrm{mmol} / \mathrm{L}$ to $35 \mathrm{mmol} / \mathrm{L}$

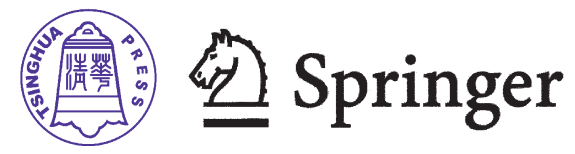


in a conventional three-electrode cell at room temperature. The silicon substrates were used as the working electrodes, and a platinum and Ag/ $\mathrm{AgCl}$ electrode served as the counter and reference electrode, respectively. Each measurement was performed with $5 \mathrm{mmol} / \mathrm{L}$ ferricyanide solution in $1 \mathrm{M} \mathrm{KCl}$ solution with a sweep rate of $40 \mathrm{mV} / \mathrm{s}$. The results of these electrochemical experiments indicated that of the three working electrode surfaces, the $\mathrm{Pt}$ nanowire film exhibits the greatest activity, due to the high electroactive surface area associated with the Pt nanowires. As expected, the patterned surface performed worse than the film due to the lower density of Pt nanowires present on the surface. It is also to be expected that the electrochemical activity of the Pt nanowire film would perform much better than bulk Pt electrodes due to the large surface area provided by the nanowire structure. This is supported by our previous work [15], in which we have shown that Pt nanowires coated on a Pt gauze had an electrochemically active surface area that is about two to three orders of magnitude higher than the pristine Pt gauze without Pt nanowire coating. To examine the response of the $\mathrm{Pt}$ nanowire film electrode to varying concentrations of ferricyanide, voltammetric curves were obtained with concentration increasing from $5 \mathrm{mmol} / \mathrm{L}$ to $35 \mathrm{mmol} / \mathrm{L}$, as shown in Fig. 5(b). The supporting electrolyte solution was $1 \mathrm{~mol} / \mathrm{L}$ $\mathrm{KCl}$ throughout and all runs were carried out with a sweep rate of $40 \mathrm{mV} / \mathrm{s}$. As the concentration increased, the oxidation and reduction peak currents increased montonically. These results verify that the Pt nanowire film supported on a silicon substrate can function as an electrode in an electrochemical cell. With regard to the reversibility of the redox couple, the observed potential peak separation $\left(\Delta E_{\mathrm{p}}\right)$ was $110 \mathrm{mV}$ for ferricyanide concentrations in the range from $5 \mathrm{mmol} / \mathrm{L}$ to $35 \mathrm{mmol} / \mathrm{L}$. This $\Delta E_{\mathrm{p}}$ value is higher than the value of $59 \mathrm{mV}$ expected for a reversible one-electron system, because of the fast scan rate of $40 \mathrm{mV} / \mathrm{s}$ used in our measurements [35]. The poly(vinylpyrrolidone) (PVP) coating on the $\mathrm{Pt}$ nanowires might also contribute to the increased separation of the redox peaks.

In summary, we have demonstrated that thin films of Pt nanowires can be directly grown onto silicon substrates, by modifying the surface with a thiolterminated silane or by creating rough features on the surface. The self-assembled monolayer of MPTMS on the silicon surface acted as strong binding sites for the polyol-formed $\mathrm{Pt}(0)$ nuclei. Once the $\mathrm{Pt}(0)$ nuclei were attached to the silicon surface, they acted as catalytic sites for the anisotropic growth of $\mathrm{Pt}$ nanowires along the $\langle 111\rangle$ direction. Similar to the MPTMS monolayer, the rough features on the silicon support acted as nucleation and binding sites due to their higher surface energies, which allowed the nucleation of $\mathrm{Pt}$ atoms and the subsequent growth of $\mathrm{Pt}$ nanowires. The principles learned from these two surface modification techniques were utilized further to demonstrate the growth of $\mathrm{Pt}$ nanowires into well-defined patterns, generated by scouring the surface of bare silicon or by etching patterns in MPTMS monolayers with a shadow mask. The results described here provides a new technique for producing thin films of $\mathrm{Pt}$ with high surface areas, and a new type of electrode that can be used as an electrochemical sensor or as an active component for applications in miniaturized fuel cells. Furthermore, the simplicity and versatility of the patterning techniques, coupled with the straightforward method of growing Pt nanowires, provide a remarkably simple method for coating multiple forms of silicon substrates with catalytically active Pt nanowires.

\section{Experimental}

Preparation of $5 \mu \mathrm{m}$ pits on silicon substrate: Beginning with a Si (100) wafer coated with $50 \AA$ of $\mathrm{Ti}$ as an adhesion layer and $1000 \AA \mathrm{Ag}$ (purchased from the Washington Technology Center, Seattle, WA), the first step was to print a self-assembled monolayer (SAM) as a protective layer on the surface of the Agcoated silicon wafer with the desired pattern. To do this, a solution of octadecanethiol (ODT, $0.06 \mathrm{~g}$ in $1000 \mathrm{~mL}$ of ethanol) was applied to a patterned poly (dimethyl siloxane) stamp (PDMS, Sylgard 184 Elastomer kit, Dow Corning, Midland, MI) with features of $5 \mu \mathrm{m}$ lines by using a cotton Q-tip and drying the stamp with a continuous stream of nitrogen for $30 \mathrm{~s}$. The dried stamp was then brought into conformal contact with the Ag surface for $10 \mathrm{~s}$. 
After removing the stamp from the surface the stamping procedure was repeated, without re-inking, and orthogonal to the previous stamping. Once the SAM was printed onto the surface, the unprotected region of Ag was selectively etched with an etching solution $(0.8 \mathrm{~g}$ of ferric nitrate and $0.23 \mathrm{~g}$ of thiourea in $100 \mathrm{~mL}$ of DI water under magnetic stirring at 300 rpm) for $4 \mathrm{~min}$, and then rinsed with DI water and dried under a continuous stream of nitrogen. After removing the $\mathrm{Ag}$, the exposed silicon was selectively etched with an etching solution $(350 \mathrm{~g} \mathrm{KOH}$ in 700 $\mathrm{mL}$ of DI water and $200 \mathrm{~mL}$ of isopropanol, under magnetic stirring at $300 \mathrm{rpm}$ and at $85^{\circ} \mathrm{C}$ ) for $4 \mathrm{~min}$, followed by rinsing with DI water and drying under nitrogen. The final step was the complete removal of the Ag layer. This was done by immersing the entire substrate into a concentrated silver etching solution (3.2 $\mathrm{g}$ of ferric nitrate and $0.92 \mathrm{~g}$ of thiourea in $100 \mathrm{~mL}$ of DI water under magnetic stirring at $300 \mathrm{rpm}$ ) for 7 min, followed by rinsing with DI water and drying.

Preparation of patterned surfaces: The scratched, checkered patterns were prepared by scouring lines across the surface of a clean silicon substrate with a diamond cutter, followed by a thorough wash with water and ethanol. The second method for generating checkered patterns required the use of copper grids (PELCO ${ }^{\circledR}$ Grids, 1GC 300 mesh), which were used as shadow masks for patterning, and MPTMS (0.5 $\mathrm{mmol} / \mathrm{L}$ in $\mathrm{MeOH}$ ) to functionalize the surface of the silicon substrate. Initially, a clean silicon wafer was functionalized with MPTMS by dip coating, and then washed immediately with copious amounts of ethanol to remove excess MPTMS, followed by drying under a continuous stream of air. Next, a copper grid was taped over the functionalized surface and the sample was plasma cleaned at $100 \mathrm{~W}$ for $10 \mathrm{~min}$. In doing this, regions in which the copper grid did not cast a shadow were cleaned by plasma, leaving a monolayer of MPTMS with a checkered pattern. After plasma etching the copper grid was carefully removed from the substrate.

Synthesis of Pt nanowires on silicon substrates: The formation of Pt nanowires on the patterned and/ or functionalized silicon substrates, typically began by injecting $4 \mathrm{~mL}$ of ethylene glycol (e. g., J. T. Baker, Lot\# A34B16) into a 3-neck flask (fitted with a reflux condenser and a teflon coated stir bar) which was heated in air at $110{ }^{\circ} \mathrm{C}$ for $30 \mathrm{~min}$. The patterned and / or functionalized silicon substrate was then added to the ethylene glycol along with $20 \mu \mathrm{L}$ of a $20 \mathrm{mmol} / \mathrm{L}$ iron species $\left(\mathrm{FeCl}_{3} \cdot 6 \mathrm{H}_{2} \mathrm{O}\right.$ or $\mathrm{FeCl}_{2} \cdot 6 \mathrm{H}_{2} \mathrm{O}$, Aldrich, predissolved in ethylene glycol). The solution was heated for an additional $30 \mathrm{~min}$ to boil off any trace amounts of water. Poly(vinylpyrrolidone) (PVP, $0.045 \mathrm{~g}$, Aldrich, $\mathrm{MW} \approx 55,000)$ and $\mathrm{H}_{2} \mathrm{PtCl}_{6}(0.033 \mathrm{~g}$, Aldrich $)$ were each dissolved separately in $1 \mathrm{~mL}$ of ethylene glycol at room temperature. These two solutions were then added simultaneously into the flask over a period of $1.5 \mathrm{~min}$. The molar ratio between $\mathrm{H}_{2} \mathrm{PtCl}_{6}$ and the repeating unit of PVP was 1:5. The reaction mixture was heated at $110{ }^{\circ} \mathrm{C}$ in air. After $18 \mathrm{~h}$, the final solution was colorless with black aggregates on the silicon substrate and at the bottom of the flask. The silicon substrate was washed thoroughly with ethanol and water to remove ethylene glycol and excess PVP.

Electrochemical measurements: Electrochemical activities were measured by cyclic voltammetry using a standard three-electrode cell at a computercontrolled Perkin Elmer Potentiostat/Galvanostat Model 283 electrochemical workstation. The sample of Pt nanowires on a silicon substrate was used as the working electrode, which was clipped to a $\mathrm{Cu}$ wire bent into a paper clip to ensure a conductive contact. A Pt wire and a $\mathrm{Ag} / \mathrm{AgCl}$ electrode served as the counter and reference electrode, respectively. Tests were performed by cycling the voltage between $0.1 \mathrm{~V}$ and $0.5 \mathrm{~V}$ in $5 \mathrm{mmol} / \mathrm{L}$ to $35 \mathrm{mmol} / \mathrm{L}$ aqueous potassium ferricyanide (Aldrich) solution at room temperature. $1 \mathrm{~mol} / \mathrm{L} \mathrm{KCl}$ (Aldrich) was used as the supporting electrolyte. The scan rate was $40 \mathrm{mV} / \mathrm{s}$.

Materials characterization: The SEM samples were prepared by placing the as-prepared silicon substrates on a carbon tape, washing with a large volume of water, and drying under ambient conditions. SEM images were taken using a Sirion XL field-emission microscope (FEI, Hillsboro, OR) operated at an acceleration voltage in the range 10$15 \mathrm{kV}$. High-resolution TEM images were performed using a JEOL JEM-2100F microscope operated at an accelerating voltage of $200 \mathrm{kV}$. Electrochemical experiments were performed with a Model 283 
Potentiostat/ Galvanostat (Advanced Measurement Technology, Inc., Oak Ridge, TN).

\section{Acknowledgments}

This work was supported in part by two research grants from the NSF (DMR-0451788) and ACS (PRF44353-AC10).

\section{References}

[1] Xiong,Y.; Wiley, B. J.; Xia, Y. Nanocrystals with unconventional shapes-A class of promising catalysts. Angew. Chem. Int. Ed. 2007, 46, 7157-7159.

[2] Girishkumar, G.; Vinodgopal, K.; Kamat, P. V. Carbon nanostructures in portable fuel cells: Single-walled carbon nanotube electrodes for methanol oxidation and oxygen reduction. J. Phys. Chem. B 2004, 108, 1996019966.

[3] Vaseashta, A.; Dimova-Malinovska, D. Nanostructured and nanoscale devices, sensors and detectors. Sci. Technol. Adv. Mat. 2005, 6, 312-318.

[4] Wang, Y.; Shah, N.; Huffman, G. P. Pure hydrogen production by partial dehydrogenation of cyclohexane and methylcyclohexane over nanotube-supported Pt and Pd catalysts. Energ. Fuel. 2004, 18, 1429-1433.

[5] Nagai, Y.; Shinjoh, H.; Yokota, K. Oxidation selectivity between $n$-hexane and sulfur dioxide in diesel simulated exhaust gas over platinum-supported zirconia catalyst. Appl. Catal. B 2002, 39, 149-155.

[6] Zhang, Z.; Liu, H.; Deng, J. A glucose biosensor based on immobilization of glucose oxidase in electropolymerized o-aminophenol film on platinized glassy carbon electrode. Anal. Chem. 1996, 68, 1632-1638.

[7] Liu, Z.; Ling, X. Y.; Su, X.; Lee, J. Y. Carbon-supported Pt and PtRu nanoparticles as catalysts for a direct methanol fuel cell. J. Phys. Chem. B 2004, 108, 8234-8240.

[8] Hyman, M. P.; Medlin, J. W. Mechanistic study of the electrochemical oxygen reduction reaction on $\mathrm{Pt}(111)$ using density functional theory. J. Phys. Chem. B 2006, 110, 15338-15344.

[9] Chen, Z.; Waje, M.; Li, W.; Yan,Y. Supportless Pt and PtPd nanotubes as electrocatalysts for oxygen-reduction reactions. Angew. Chem. Int. Ed. 2007, 46, 4060-4063.

[10] Chen, J.; Herricks, T.; Geissler, M.; Xia, Y. Single-crystal nanowires of platinum can be synthesized by controlling the reaction rate of a polyol process. J. Am. Chem. Soc. 2004, 126, 10854-10855.

[11] Chen, J.; Herricks, T.; Xia, Y. Polyol synthesis of platinum nanostructures: Control of morphology through the manipulation of reduction kinetics. Angew. Chem. Int. Ed. 2005, 44, 2589-2592.

[12] Narayanan, R.; El-Sayed, M. A. Catalysis with transition metal nanoparticles in colloidal solution: Nanoparticle shape dependence and stability. J. Phys. Chem. B 2005, 109, 12663-12676.

[13] Teng, X.; Liang, X.; Maksimuk, S.; Yang, H. Synthesis of porous platinum nanoparticles. Small 2006, 2, 249-253.

[14] Song, H.; Kim, F.; Connor, S.; Somorjai, G. A.; Yang, P. Pt nanocrystals: Shape control and Langmuir-Blodgett monolayer formation. J. Phys. Chem. B 2004, 109, 188193.

[15] Lee, E. P.; Peng, Z.; Cate, D. M.; Yang, H.; Campbell, C. T.; Xia, Y. Growing Pt nanowires as a densely packed array on metal gauze. J. Am. Chem. Soc. 2007, 129, 1063410635.

[16] Lee, E. P.; Chen, J.; Yin, Y.; Campbell, C. T.; Xia, Y. Pdcatalyzed growth of Pt nanoparticles or nanowires as dense coatings on polymeric and ceramic particulate supports. Adv. Mater. 2006, 18, 3271-3274.

[17] Xiao, Z.; Yan, G.; Feng, C.; Chan, P. C. H.; Hsing, I. M. A silicon-based fuel cell micro power system using a microfabrication technique. J. Micromech. Microeng. 2006, 16, 2014-2020.

[18] Chu, K. L.; Shannon, M. A.; Masel, R. I. An improved miniature direct formic acid fuel cell based on nanoporous silicon for portable power generation. J. Electrochem. Soc. 2006, 153, A1562-A1567.

[19] Kelley, S. C.; Deluga, G. A.; Smyrl, W. H. A miniature methanol/air polymer electrolyte fuel cell. Electrochem. Solid-State Lett. 2000, 3, 407-409.

[20] Kelley, S. C.; Deluga, G. A.; Smyrl, W. H. Miniature fuel cells fabricated on silicon substrates. AlChE J. 2002, 48, 1071-1082.

[21] Aravamudhan, S.; Rahman, A. R. A.; Bhansali, S. Porous silicon based orientation independent, self-priming micro direct ethanol fuel cell. Sens. Actuators, A 2005, 123124, 497-504.

[22] Jiang, X.; Herricks, T.; Xia, Y. CuO nanowires can be synthesized by heating copper substrates in air. Nano Lett. 2002, 2, 1333-1338.

[23] Chai, J.; Wang, D.; Fan, X.; Buriak, J. M. Assembly 
of aligned linear metallic patterns on silicon. Nat. Nanotechnol. 2007, 2, 500-506.

[24] Mieszawska, A. J.; Zamborini, F. P. Gold nanorods grown directly on surfaces from microscale patterns of gold seeds. Chem. Mater. 2005, 17, 3415-3420.

[25] Duan, G.; Cai, W.; Luo, Y.; Li, Y.; Lei, Y. Hierarchical surface rough ordered Au particle arrays and their surface enhanced Raman scattering. Appl. Phys. Lett. 2006, 89, 181918.

[26] Huang, Y.; Duan, X.; Wei, Q.; Lieber, C. M. Directed assembly of one-dimensional nanostructures into functional networks. Science 2001, 291, 630-633.

[27] Wei, Z.; Mieszawsk, A. J.; Zamborini, F. P. Synthesis and manipulation of high aspect ratio gold nanorods grown directly on surfaces. Langmuir 2004, 20, 4322-4326.

[28] Gao, X.; Tam, K.; Yu, K. M. K.; Tsang, S. C. Synthesis and characterization of thiol-capped FePt nanomagnetic porous particles. Small 2005, 1, 949-952.

[29] Brito, R.; Rodriguez, V. A.; Figueroa, J.; Cabrera, D. R. Adsorption of 3-mercaptopropyltrimethoxysilane and 3-aminopropyltrimethoxysilane at platinum electrodes. J. Electroanal. Chem. 2002, 520, 47-52.
[30] Laiho, T.; Lukkari, J.; Meretoja, M.; Laajalehto, K.; Kankare, J.; Leiro, J. A. Chemisorption of alkyl thiols and S-alkyl thiosulfates on Pt(111) and polycrystalline platinum surfaces. Surf. Sci. 2005, 584, 83-89.

[31] Laiho, T.; Leiro, J. A.; Lukkari, J. XPS study of irradiation damage and different metal-sulfur bonds in dodecanethiol monolayers on gold and platinum surfaces. Appl. Surf. Sci. 2003, 212-213, 525-529.

[32] Gouldstone, A.; Van Vliet, K. J.; Suresh, S. Nanoindentation: Simulation of defect nucleation in a crystal. Nature 2001, 411, 656.

[33] Brune, H. Microscopic view of epitaxial metal growth: Nucleation and aggregation. Surf. Sci. Rep. 1998, 31 , 125-229.

[34] Niederhauser, T. L.; Jiang, G.; Lua, Y. Y.; Dorff, M. J.; Woolley, A. T.; Asplund, M. C.; Berges, D. A.; Linford, M. R. A new method of preparing monolayers on silicon and patterning silicon surfaces by scribing in the presence of reactive species. Langmuir 2001, 17, 5889-5900.

[35] Ramesham, R. Voltammetric study at the polycrystalline diamond grown over a graphite electrode material. Thin Solid Films 1999, 339, 82-87. 